\title{
CONSTRUCCIÓN Y EVALUACIÓN DE UNA COCINA/HORNO SOLAR TIPO CAJA
}

\author{
Hugo Torres Muro'; Carlos Polo Bravo'; \\ Orlando López Cornejo ${ }^{2}$; Boris Milla Taco ${ }^{3}$
}

\section{$\checkmark$ \\ RESUMEN}

Una de las alternativas de solución al problema de la contaminación ambiental y la deforestación es el uso masivo de tecnologías que aprovechen la energía solar como fuente de energía primaria; entre estas tecnologías se encuentran las cocinas solares, las que se han desarrollado y probado con éxito en diferentes partes del mundo. En nuestro país aun se están realizando los primeros programas de investigación y difusión.

Este proyecto tiene como objetivo demostrar que las cocinas solares tipo caja pueden ser usadas en nuestra región con éxito. Para evaluar nuestra cocina solar se ha aplicado un procedimiento internacional estandarizado. Los resultados obtenidos han sido satisfactorios, llegándose a obtener temperaturas por encima de los $100^{\circ} \mathrm{C}$ en la cámara de cocción, lo que nos permitirá preparar algunos alimentos de los más comunes en la región.

\section{ABSTRACT}

One of the solution alternatives to the problem of the environmental contamination and the deforestation is the massive use of technologies that you/they take advantage of the solar energy as source of primary energy; among these technologies they are the solar kitchens, of which have been developed and proven with success, several types in different parts of the world. In our country they are even being carried out the first investigation programs and diffusion.

This project has as objective to demonstrate that the kitchens solar type box can be used in our region with success. To evaluate our solar kitchen a standardized international procedure it has been applied. The obtained results, they have been satisfactory, being ended up obtaining temperatures above the $100^{\circ} \mathrm{C}$ in the cooking camera what allowed to get ready some foods of the most common in the region.

\section{INTRODUCCIÓN}

La energía es sin duda uno de los factores más importantes en la vida del hombre, gracias a ella la humanidad ha podido dominar la naturaleza y llegar a su actual grado de desarrollo científico y tecnológico. Sin embargo, la estructura del actual sistema energético permite predecir la imposibilidad de su sostenimiento a largo plazo, como base para un desarrollo sostenible de la humanidad; por ello resulta imprescindible encontrar alternativas de solución viables que nos permitan afrontar este problema con posibilidades de éxito para todas las regiones del mundo.

1 Licenciado en Fisica.

2 Bach. en Minas.

3 Bach. en Fisica con mención en Electrónica. 
La Comisión Mundial del Medio Ambiente y del Desarrollo analizó, por encargo de la Asamblea General de las Naciones Unidas, las posibles alternativas para solucionar la crisis global de la energía. En su informe correspondiente, concluye: “...La eficiencia energética sólo permite ganar tiempo mientras se desarrollan medios de bajo consumo de energía basados en fuentes renovables, que son las que deberán constituir el fundamento de la estructura energética mundial durante el siglo XXI. La mayoría de estas fuentes son actualmente problemáticas, pero si se innova en el desarrollo pueden suministrar el mismo volumen de energía primaria que es consumida actualmente en el planeta..."(1).

La reforestación, el uso de fuentes renovables de energía y la conservación de combustibles, son los tres métodos que ofrecen soluciones posibles a la crisis global de la energía en general y a la crisis de la leña en particular. La energía solar como fuente de energía que sustituya a la leña representa una alternativa importante de solución. En el Perú, en las zonas rurales, el uso de la leña como fuente primaria de energía representa una fracción muy importante de todas las necesidades energéticas de la población; y su escasez es cubierta en muchos casos con el uso del estiércol y querosene, lo que perjudica la agricultura y la economía de dichas poblaciones.

El potencial energético solar en la región Tacna permite el aprovechamiento de la energía solar como fuente de energía primaria, por lo tanto, es posible aplicar diferentes tecnologías solares ya probadas, como las cocinas solares tipo caja o tipo parabólica para obtener beneficios prácticos y económicos. Se utilizan principalmente, para cocinar alimentos, pasteurizar agua, hornear panes, tortas, galletas 0 esterilizar instrumentos.

Son numerosos los factores que favorecen que las cocinas solares sean asequibles para las personas comunes; con un conocimiento de los principios básicos de la energía solar y un acceso a materiales simples, como el cartón, el papel de aluminio y el vidrio, se puede construir una cocina solar eficaz.

\subsection{LA CRISIS GLOBAL DE LA ENERGIA Y SUS CONSECUENCIAS}

- En la actualidad, la energía que consume el hombre proviene aproximadamente en un $80 \%$ de los combustibles fósiles como el carbón, el petróleo y el gas natural, cuya utilización masiva y sin capa de ozono y la acidificación del ciclo del agua. (2)

- La distribución del consumo de energía es sumamente desigual; los países altamente industrializados consumen 80 veces más que los países "pobres», los cuales a su vez se encuentran en limites inferiores a los permitidos para la supervivencia. De ello se desprende que un cuarto de la población mundial consume las tres cuartas partes de la energía primaria del mundo.

- Las políticas de subvención de los precios de los combustibles, lejos de contribuir al incremento de la eficiencia energética, fomentaron el despilfarro, han permitido la instalación de una industria ineficiente que, en poco tiempo, ha visto disminuir su competitividad, contribuyendo a aumentar la enorme desigualdad entre ricos y pobres.

- Entre otras cosas, esta situación ha conducido a la crisis de la leña y, en consecuencia, a la deforestación de los bosques, que es una forma de crisis de la energía muy particular en los paises en desarrollo. Para los países «más pobres» del mundo, la madera como fuente primaria de energía es vital; nueve de cada diez personas dependen de la leña como fuente principal de combustible; 1500 millones de personas en el mundo cocinan y se calientan con madera. Esto quiere decir que del 50 al $70 \%$ de toda la madera usada en la Tierra termina bajo alguna olla de cocinar. (2)

- A medida que los precios de la leña suben, las personas se ven obligadas a usar combustibles derivados del petróleo, lo cual lleva a una mayor presión sobre los bosques y un cambio al estiércol. La quema de estiércol agota la fertilidad de los suelos, baja el rendimiento de las cosechas y contribuye al esparcimiento del hambre y la migración de la gente y los rebaños a tierras más habitables o a ciudades ya superpobladas. La migración obligada trae consigo la desertificación, sucumbiendo los terrenos para el cultivo y el pastoreo. Cuando la leña de fuentes cercanas se termina, ellos cambian al carbón vegetal, que resulta más barato transportarla a largas distancias, sin embargo, el cocinar con carbón vegetal representa desde 1,5 a 3,0 veces más gasto de madera. 


\subsection{FUNCIONAMIENTO DE UNA COCINA/ HORNO SOLAR TIPO CAJA}

La cocina solar tipo caja más simple y barata consiste en una caja aislada, con una ventana de plástico o vidrio. La ventana actúa como una trampa para retener el calor de la energía solar por efecto invernadero. La radiación solar atraviesa la ventana de vidrio e impide que la radiación que se refleja de las paredes y las ollas se escape fuera de la cocina, calentando la placa negra, el aire y la olla negra que se encuentra en la cámara de cocción. Las paredes de la cocina se revisten con papel aluminio de forma que refleje toda la radiación hacia las ollas pintadas de negro. La placa de metal pintada de negro lleva ei calor a las ollas, por conducción. Para aumentar la radiación solar entrante, pueden usarse reflectores, los cuales deben ubicarse para reflejar la radiación solar hacia el interior de la caja. La caja debe estar aislada lo mejor posible, para minimizar el flujo de aire caliente al exterior.

Debido a la acción de la cocina solar, el calor que es absorbido por la plancha y las ollas de metal pintadas de negro, es transferido por conducción a través de esos materiales para calentar y cocinar los alimentos. En las figuras 1 y 2 , respectivamente, se muestra un esquema muy simplificado de una cocina solar tipo caja y la forma en que se produce el efecto invernadero para este caso.(7)

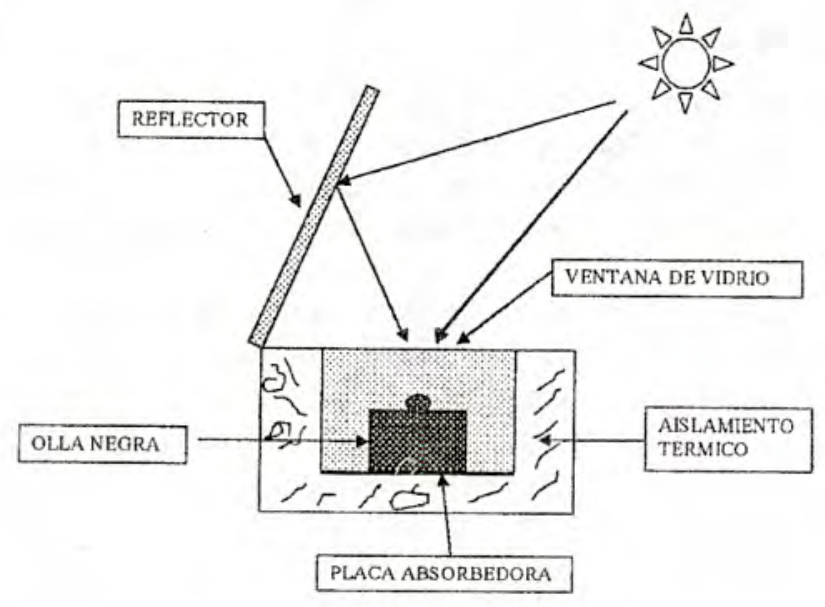

Figura 1. Esquema simplificado que muestra cómo funciona una cocina solar tipo caja.(5)

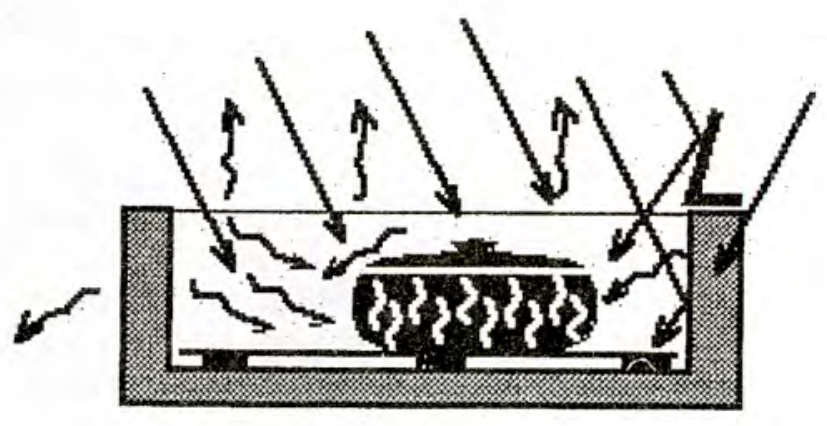

Figura 2. Esquema que muestra cómo se produce el efecto invernadero en una cocina/horno solar tipo caja (7).

\subsection{ALMACENAMIENTO Y PÉRDIDAS DE CALOR}

El buen almacenamiento de calor dentro de la caja se debe a los materiales con los cuales se ha construido la cocina solar, principalmente el aislante térmico, el cual evitará las pérdidas de calor por las paredes y la parte inferior de la caja; asimismo, se puede colocar dos vidrios en lugar de uno solo para reducir las pérdidas de calor por convección.

La Segunda Ley de la Termodinámica plantea que el calor siempre se transfiere de un cuerpo o sustancia caliente a otra mas fría. El calor dentro de una cocina solar se pierde por tres mecanismos físicos que son; por conducción, radiación y convección (8).

- POR CONDUCCIÓN, el calor dentro de una cocina solar se pierde cuando viaja a través de las moléculas de las hojas de aluminio, el vidrio, el cartón, el aire y el aislamiento, hacia el aire fuera de la caja. La placa absorbente calentada por el sol conduce el calor a la parte inferior de las cacerolas. Para prevenir la pérdida de este calor vía conducción a través de la parte inferior de la cocina, la placa absorbente se eleva de la parte inferior utilizando pequeños espaciadores aislantes.

- POR RADIACIÓN, todo cuerpo que se encuentra a una determinada temperatura, emite radiación en el rango del infrarrojo, este calor se propaga a través del aire. La mayor parte del calor radiante que se despide de las ollas calientes dentro de una cocina solar se refleja desde el papel de aluminio y del cristal nuevamente a las ollas y a la bandeja inferior. Aunque los vidrios son opacos a la radiación infrarroja, un poco de ésta escapa al exterior. 
- POR CONVENCIÓN, se pierde calor en las cocinas solares cuando el aire más frío que el vidrio de la ventana caliente pasa sobre ella y arrastra el calor de las moléculas de aire en contacto directo con el vidrio o, simplemente, estas moléculas de aire caliente se elevan al disminuir su densidad y son reemplazadas por otras más frías, lo mismo sucede cuando las moléculas del aire entran y salen de la caja a través de las rendijas.

\subsection{ANÁLISIS TEÓRICO DE UNA COCINA SOLAR TIPO CAJA}

El análisis teórico que presentamos es para una cocina solar tipo caja rectangular, provista de una placa reflectora y una placa plana absorbedora, pintada de negro, como la que se muestra en la figura 1. Este tipo de análisis se puede aplicar a otros diseños típicos.(5).

\section{- Energia Aprovechable}

La energía solar (irradiación) aprovechable por una superficie horizontal varía predeciblemente con el día y la hora, con la rotación y órbita de nuestro planeta. Pero existen varios factores impredecibles que limitan el aprovechamiento de esta energía como son, la humedad, polución atmosférica y los cielos nublados. Por esta razón, la irradiancia o potencia solar incidente por unidad de área, sobre una superficie horizontal, debe ser medida durante los experimentos.

La energía solar aprovechable por una cocina solar con ventana horizontal es reforzada por la contribución del reflector menos la sombra que proyecta al interior. La contribución del reflector es una función de su ángulo con respecto a la radiación incidente y la ventana, la reflactancia, la composición de la radiación y la claridad del cielo. Teniendo en cuenta varios componentes, la ecuación resultante/ $5 /$ que describe la energía total aprovechable, $Q_{a}$, está dada por:

$Q_{a}=A_{a}\left\{I_{b}+\left[\rho_{s} I_{b, n} \operatorname{sen}\left(\theta_{z}\right)\right]+\left[I_{d}\left(F_{a, s}+F_{a^{\prime}, t}\right]\right\} \ldots \ldots . .1\right.$

\section{Donde:}

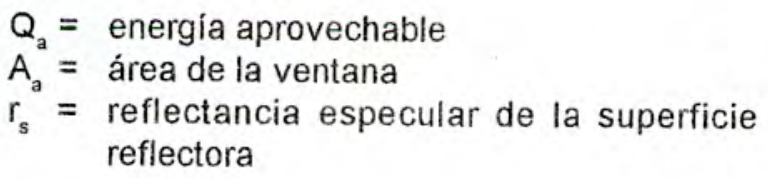

$\mathrm{I}_{\mathrm{b}}=$ radiación directa

$\mathrm{I}_{\mathrm{b}, n}=$ radiación directa normal

$\theta_{\mathrm{z}}=$ ángulo cenital

$I_{\mathrm{d}}=$ radiación difusa

$\mathrm{F}_{\mathrm{A}, \mathrm{S}}=$ Factor de forma, ventana al cielo $=0,77$

$F_{A, R}=$ Factor de forma, reflector a ventana $=0,23$, $q=90^{\circ}$

$\rho=$ reflectancia general de la superficie reflectora

A su vez algunos de estos componentes tienen su expresión que en todo caso se puede determinar fácilmente, recurriendo a la referencia (5).

Un aumento en la temperatura del fluido de prueba(de los alimentos) es considerado como energía útil. Esta energía disponible no se usa al 100 $\%$ en cocinar los alimentos, porque una parte es perdida por diversos mecanismos, ya indicados. Holman/6/ nos proporciona una ecuación que describe el calor almacenado o calor útil, dado por:

$Q_{u}=m C_{p} D T / \Delta t$ 2

Donde $\mathrm{m}$ es la masa de olla y placa plana, $\mathrm{Cp}$ el calor específico de los metales y no metales, y $\Delta T$ el cambio de temperatura en un de tiempo $\Delta t$.

La eficiencia de la cocina,e, es la energía útil ganada sobre la energia total disponible durante el periodo de precalentamiento:

$\varepsilon=Q u / Q a$ 3

\section{MATERIALES Y MÉTODO}

\subsection{MATERIALES}

- 01 vidrio común de ventana de $50 \times 60 \mathrm{~cm}$

- 01 caja exterior de $56 \times 66 \times 25 \mathrm{~cm}$

01 caja interior de $46 \times 56 \times 20 \mathrm{~cm}$

01 tapa de $71 \times 61 \times 8 \mathrm{~cm}$

- 08 soportes inferiores de la caja interior, se hacen con pedazos de cartón colado o triplay, de manera que formen cubitos de $4 \mathrm{~cm}$ de alto

- 04 sellos contra la humedad, si las cajas no tienen "faldillas"

01 rollo de papel de aluminio de $10 \mathrm{~m} \times 30 \mathrm{~cm}$

- 02 tubos de silicona

01 litro de cola sintética basada en agua

02 ollas pintadas de negro con tapas de acuerdo con el tamaño de la cocina 
- 01 plancha de metal (latón, aluminio, cobre o bronce de $44 \times 54 \mathrm{~cm}$

- 01 soporte de madera de $70 \mathrm{~cm}$ de largo

01 lata de pintura negro-mate (para pizarra) sin aceite ni resina

Papel de bolsa de azúcar, para forrar el exterior de la caja

01 aplicador de silicona

\subsection{CONSTRUCCIÓN DE LA COCINA SOLAR TIPO CAJA/8/}

Paso 1. Usando cajas con las medidas especificadas o muy aproximadas, nos aseguramos que el espacio que debe quedar entre la caja interior y exterior debe ser de 3 a $4 \mathrm{~cm}$, luego ajustamos las alturas de las cajas, doblando las paredes de las cajas para que midan los $20 \mathrm{~cm}$ la caja interior y $25 \mathrm{~cm}$ la caja exterior. Si la caja tiene faldillas, se doblan cuidadosamente una sobre otra. Para hacer la tapa se consigue una pieza plana de cartón que mida 15 $\mathrm{cm}$ más de largo y ancho que la caja exterior. Se pone dicha pieza sobre la caja y se doblan los rebordes sobre la caja para que quede bien ajustada. Cortar y doblar los faldillos de las esquinas.

Paso 2. Cortamos el papel aluminio de acuerdo con las dimensiones deseadas, lo encolamos y pegamos en las caras laterales y fondo interno de la caja exterior, en ambos lados de las paredes y fondo de la caja interior, en la cara interna de la tapa y en las faldillas que están en la caja interna. Los soportes de madera de la caja pequeña de $4 \mathrm{~cm}$ de espesor, $20 \mathrm{~cm}$ de longitud y $4 \mathrm{~cm}$ de ancho, los hemos colocado en el fondo de la caja grande, por dentro. Colocamos el aislante térmico, en nuestro caso es lana de vidrio revestido con papel aluminio; colocamos la caja interior y rellenamos los espacios entre las cajas con el mismo material aislante.

Paso 3. Con la tapa ya preparada anteriormente haremos la ventana y el reflector. Para ello dibujamos un rectángulo en el interior de la tapa, pero un poco más pequeño que el vidrio que vamos a colocar luego. Cortamos sólo tres lados del rectángulo, uno largo y dos cortos, dejando el largo restante para doblar el cartón que servirá como reflector. Para hacer el soporte del reflector utilizamos una varilla delgada de madera. En los bordes internos del vidrio colocamos en forma uniforme y fina la silicona. Luego colocamos el vidrio en la parte interior de la tapa y quedará lista la ventana transparente.

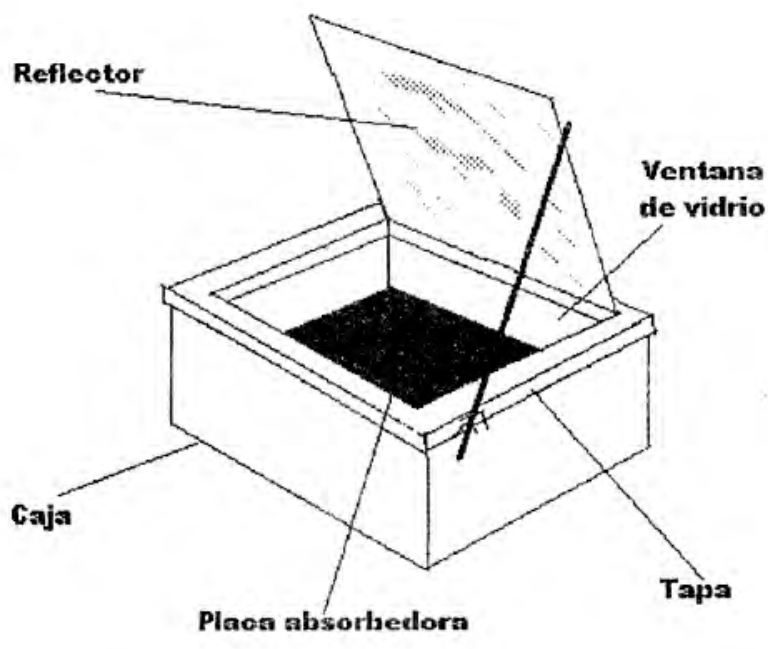

Figura 3. Esquema que muestra la cocina/horno solar construida en el CERT-UNJBG. Se pueden observar sus partes externas más importantes.

\subsection{MÉTODO DE EVALUACIÓN}

\section{- Equipos e Instrumentos}

- 01 Piranómetro Moll - Gorczynsky de la Kipp and Zonen, de sensibilidad espectral comprendido en el rango entre 0,3 y $3 \mathrm{~mm}$, con un tiempo de respuesta de 5 segundos y una constante de calibración de $12,35 \times 10^{-6} \mathrm{mV} / \mathrm{Wm}^{-2}$. Con este instrumento hemos medido la variación de la irradiancia solar sobre una superficie horizontal.

- 05 Termocúplas de níquel - cromo - níquel, como sensores de temperatura, ubicadas en diferentes partes de la cocina solar.

- 01 Registrador Multicanal computarizado marca Grant, con capacidad de memoria para 50000 datos.

- 01 Anemómetro de cazoletas de fabricación alemana, con una constante de calibración igual a $0,02 \times 10^{3} \mathrm{~ms}^{-1} / \mathrm{mV}$. Nos permite determinar la variación de la velocidad del viento a través del tiempo.

- 01 olla de aluminio común, pintada de negra sin brillo y con tapa. 


\section{- Procedimiento de Evaluación}

Hemos aplicado el procedimiento propuesto por H. P. Garg, del Centro de Estudios de la Energía, del Instituto de Tecnología de la India, Nueva Delhi, presentado en la Segunda Conferencia Mundial de Cocinas Solares, de Julio de 1994, realizada en Costa Rica./9/. El procedimiento supone que las cubiertas del vidrio tienen una transmisión óptica buena y la cocina tiene un bajo factor de pérdidas globales de calor. Consiste en realizar medidas de la temperatura de placa de la cocina, de la cámara, temperatura ambiente y velocidad del aire y radiación solar global sobre una superficie horizontal, para diferentes condiciones de operación. Considerándose, entre ellas, la evaluación con reflector y sin reflector, con carga y sin carga, se considera como carga un litro de agua, que puede ser destilada. Las mediciones realizadas nos han permitido datos, entre los cuales tenemos:

- Temperatura Ambiente (Tamb), bajo sombra, cada minuto del dia.

- Temperatura de Placa (Tp) de la cocina solar, cada minuto del día.

- Temperatura de la Cámara (Tc) de cocción, cada minuto del día.

- Temperatura del agua (Ta) contenida en la olla, cada minuto del día.

- Velocidad del viento( $(V v)$ cada minuto del día.

- Irradiación global para una superficie horizontal (G), cada minuto del día.

\section{RESULTADOS}

- De acuerdo con la Tabla $N^{\circ} 01$, la temperatura máxima de placa $\mathrm{Tp}=112,4^{\circ} \mathrm{C}$ sin carga y sin reflector se obtuvo el día 08 de febrero del 2000 , para una irradiancia de $979,9 \mathrm{~W} / \mathrm{m}^{2}$; en un tiempo de 7 horas.

- De acuerdo con la Tabla $\mathrm{N}^{\circ} 02$, la temperatura máxima de placa $\mathrm{Tp}=137,8^{\circ} \mathrm{C}$, sin carga y con reflector se obtuvo el día 09 de febrero del 2000 , para una irradiancia de $997,8 \mathrm{~W} / \mathrm{m}^{2}$, en un tiempo de 03 horas.
- De acuerdo con la Tabla $N^{0} 03$, la temperatura máxima de placa $\mathrm{Tp}=96,2^{\circ} \mathrm{C}$, con carga y con reflector se obtuvo el día 07 de marzo del 2000 , para una irradiancia de $540,1 \mathrm{~W} / \mathrm{m}^{2}$, en un tiempo de 04 horas, la temperatura de la cámara alcanzó una temperatura máxima de $87,54^{\circ} \mathrm{C}$, y la del agua fue de $86,4^{\circ} \mathrm{C}$.

\section{DISCUSIÓN}

- En este proyecto se presenta un procedimiento simple y de bajo costo para construir una cocina/horno solar de buen rendimiento, cuyos parámetros físicos identificados son la temperatura de placa, la temperatura de la cámara de cocción y la radiación solar incidente.

- Los resultados obtenido de la evaluación térmica de la cocina solar nos permiten predecir que se puede cocinar la mayor parte de los alimentos más consumidos en nuestra región y que se puede utilizar para otros fines como la pasteurización del agua.

- La evaluación de la cocina/horno solar no ha concluido con este proyecto, aún queda una evaluación técrica del balance energético para determinar las pérdidas de calor a fin de mejorar su eficiencia.

\section{CONCLUSIONES}

1. La hipótesis planteada ha sido contrastada y demostrada su validez en el sentido de que la cocina/ horno solar tipo caja es una tecnología para el aprovechamiento de la energía solar factible de ser aplicada en Tacna, particularmente en las zonas rurales.

2. El uso de las cocinas solares en las zonas rurales de la región Tacna podría contribuir significativamente a reducir la deforestación, constituyéndose en una alternativa práctica y económica, para sustituir la leña y los combustibles fósiles como fuentes de energía primaria. 


\section{REFERENCIAS BIBLIOGRÁ.FICAS}

(1) Consejo Mundial de la Energía, 1998. $17^{\circ}$ Congreso del C. M. E.: Conclusiones y Recomendaciones, Houston, USA.

(2) G.T.Z. y GATE, 1985. Estufas para Ahorrar Combustible, Friedr, Vieweg y Sohn Handeldruckerei, Eschborn, República Federal de Alemania.

(3) European Committe for Solar Cooking Research(ECSCR), 1993. Third Workshop of Solar Cooker Test Procedure, Eschborn, Germany, 1993

(4) ECSCR, 1993. Second International Solar Cooker Test, Almeria, España.
(5) Funk, P.A., 1992. Analysis of a Solar Box Cooker for East Africa, Thesis for the degree of Master of Since, Minnesota, USA.

(6) Holman, J.P., 1981. Heat Transfer, $5^{\text {th }}$. Edición, Mc. Graw Hill Book Co., New York.

(7) Principios de Diseño de la Cocina Solar, 1998. http:// www2uji.es/cyer/internatura/estudios/energías/ccsolar htm/

(8) Solar Cooker International, 1994. Diseminando la Cocina Solar: Guia del Líder, Sacramento, USA.

(9) Garg, H. P., 1994. Solar Cooker Testing Procedure, Second Worid Conference on Solar Cookers: Use and Technology, Heredia, Costa Rica.

\section{ANEXOS}

Tabla 1. Valores promedios horarios de las temperaturas de placa Tp, Temperatura ambiente Tamb, velocidad del viento Vv $y$ radiación solar global $G$, sin reflector y sin carga. 08/02/ 2000 .

\begin{tabular}{|c|c|c|c|c|c|c|c|}
\hline DIA & TIEMPO $(\mathrm{h})$ & $\mathrm{Tp}(\mathrm{oC})$ & $\mathrm{Tamb}(\mathrm{oC})$ & $V v(\mathrm{mV})$ & $V v(\mathrm{~m} / \mathrm{s})$ & $G(\mathrm{mV})$ & $\mathrm{G}\left(\mathrm{W} / \mathrm{m}^{2}\right)$ \\
\hline $08 / 02 / 00$ & $06: 00$ & 16.7 & 16.8 & 0 & 0 & 0.2 & 161.94 \\
\hline $08 / 02 / 00$ & $06: 59$ & 17.357 & 17.405 & 0.000 & 0 & 0.627 & 50.768 \\
\hline $08 / 02 / 00$ & $08: 00$ & 19.659 & 19.675 & 0.037 & 0.74 & 2.288 & 185.259 \\
\hline $08 / 02 / 00$ & $09: 01$ & 22.444 & 22.302 & 0.067 & 1.34 & 7.044 & 570.353 \\
\hline $08 / 02 / 00$ & $10: 02$ & 24.887 & 24.620 & 0.071 & 1.42 & 9.464 & 766.300 \\
\hline $08 / 02 / 00$ & 11.02 & 25.118 & 26.487 & 0.100 & 2 & 11.127 & 900.953 \\
\hline $08 / 02 / 00$ & $12: 02$ & 55.146 & 27.390 & 0.115 & 2.3 & 12.011 & 972.531 \\
\hline $08 / 02 / 00$ & $13: 03$ & 112.372 & 28.623 & 0.179 & 3.58 & 12.103 & 979.980 \\
\hline $08 / 02 / 00$ & $14: 03$ & 63.113 & 29.058 & 0.227 & 4.54 & 11.215 & 908.079 \\
\hline $08 / 02 / 00$ & $15: 04$ & 44.872 & 28.502 & 0.204 & 4.08 & 9.434 & 763.871 \\
\hline $08 / 02 / 00$ & $16: 05$ & 36.872 & 27.043 & 0.195 & 3.9 & 6.944 & 562.256 \\
\hline $08 / 02 / 00$ & $17: 06$ & 32.328 & 25.125 & 0.180 & 3.6 & 4.002 & 324.042 \\
\hline $08 / 02 / 00$ & $18: 07$ & 29.097 & 22.841 & 0.162 & 3.24 & 0.797 & 64.533 \\
\hline $08 / 02 / 00$ & $19: 08$ & 26.385 & 21.070 & 0.104 & 2.08 & 0.007 & 0.567 \\
\hline $08 / 02 / 00$ & $19: 54$ & 24.554 & 20.078 & 0.058 & 1.16 & 0.000 & 0.000 \\
\hline
\end{tabular}


Gráfica 1. En la gráfica se observa que la temperatura de placa de la cocina/horno solar alcanza su temperatura media horaria máxima de $112^{\circ} \mathrm{C}$ después de aproximadamente 1:30 horas después de haber sido expuesta al sol, teniendo en cuenta que la condición de operación es sin reflector y sin carga. Tp es la temperatura de placa y Tamb es la temperatura del medio ambiente.

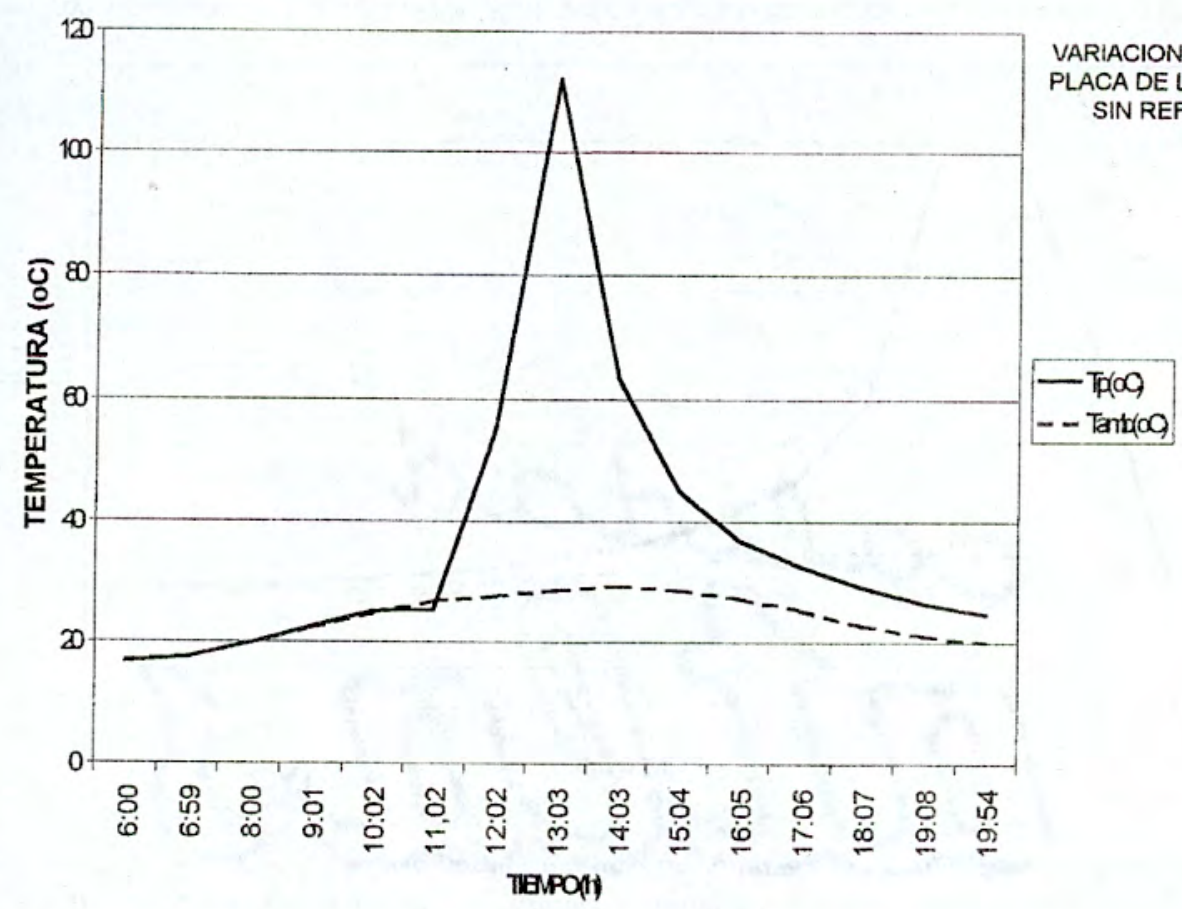

Tabla 2. Valores promedios horarios de las temperaturas de placa Tp, temperatura ambiente Tamb, velocidades del viento Vy y radiación solar global, con un reflector pero sin carga, 09/02/2 000, nos dan como resultado que la temperatura promedio horaria máxima de calentamiento de la placa absorbedora de la cocina solar es de $137^{\circ} \mathrm{C}$, con lo cual se demuestra que la inclusión de un reflector aumenta la concentración de calor en el interior de la cocina, lo cual nos va a permitir disminuir el tiempo de cocción de los alimentos.

\begin{tabular}{|c|c|c|c|c|c|c|c|}
\hline Dia & Tiempo(h) & $T p(0 \mathrm{C})$ & Tamb(oC) & $V v(\mathrm{mV})$ & $V v(\mathrm{~m} / \mathbf{s})$ & $G(\mathrm{mV})$ & $G\left(W / \mathrm{m}^{2}\right)$ \\
\hline $09 / 02 / 00$ & $9: 56$ & 24.300 & 26.500 & 0.180 & 3.600 & 10.400 & 842.088 \\
\hline $09 / 02 / 00$ & $10: 53$ & 86.747 & 26.905 & 0.098 & 1.960 & 10.978 & 888.889 \\
\hline $09 / 02 / 00$ & $11: 54$ & 137.769 & 29.779 & 0.136 & 2.720 & 6.079 & 492.217 \\
\hline $09 / 02 / 00$ & $12: 55$ & 136.036 & 30.372 & 0.179 & 3.580 & 12.323 & 997.793 \\
\hline $09 / 02 / 00$ & $13: 56$ & 70.625 & 30.723 & 0.222 & 4.440 & 11.559 & 935.932 \\
\hline $09 / 02 / 00$ & $14: 57$ & 49.102 & 30.005 & 0.207 & 4.140 & 9.780 & 791.887 \\
\hline $09 / 02 / 00$ & $15: 58$ & 39.146 & 28.333 & 0.243 & 4.860 & 7.372 & 596.911 \\
\hline $09 / 02 / 00$ & $16: 59$ & 33.585 & 26.552 & 0.181 & 3.620 & 4.354 & 352.543 \\
\hline $09 / 02 / 00$ & $18: 00$ & 29.964 & 24.323 & 0.160 & 3.200 & 0.931 & 75.383 \\
\hline $09 / 02 / 00$ & $19: 01$ & 27.113 & 22.661 & 0.119 & 2.380 & 0.028 & 2.267 \\
\hline $09 / 02 / 00$ & $20: 02$ & 24.982 & 21.423 & 0.063 & 1.260 & 0.000 & 0.000 \\
\hline $09 / 02 / 00$ & $21: 03$ & 23.623 & 20.520 & 0.037 & 0.740 & 0.000 & 0.000 \\
\hline $09 / 02 / 00$ & $22: 04$ & 22.689 & 20.161 & 0.010 & 0.200 & 0.000 & 0.000 \\
\hline $09 / 02 / 00$ & $23: 05$ & 22.103 & 19.628 & 0.010 & 0.200 & 0.000 & 0.000 \\
\hline $09 / 02 / 00$ & $23: 59$ & 21.631 & 19.326 & 0.029 & 0.580 & 0.000 & 0.000 \\
\hline
\end{tabular}


Gráfica 2. Esta gráfica nos muestra qué tan rápido la placa absorbedora llega a su temperatura máxima. Se observa también la relación que existe con la temperatura ambiente y la gran diferencia que se establece entre sus valores máximos.

CURVA DE CALENTAMIENTO/ENFRIAMIENTO DE PLACA

DE LA COCINA /HORNO SOLAR CON REFLECTOR Y SIN CARGA EN FUNCIÓN DEL. TIEMPO (T/09/02/00)

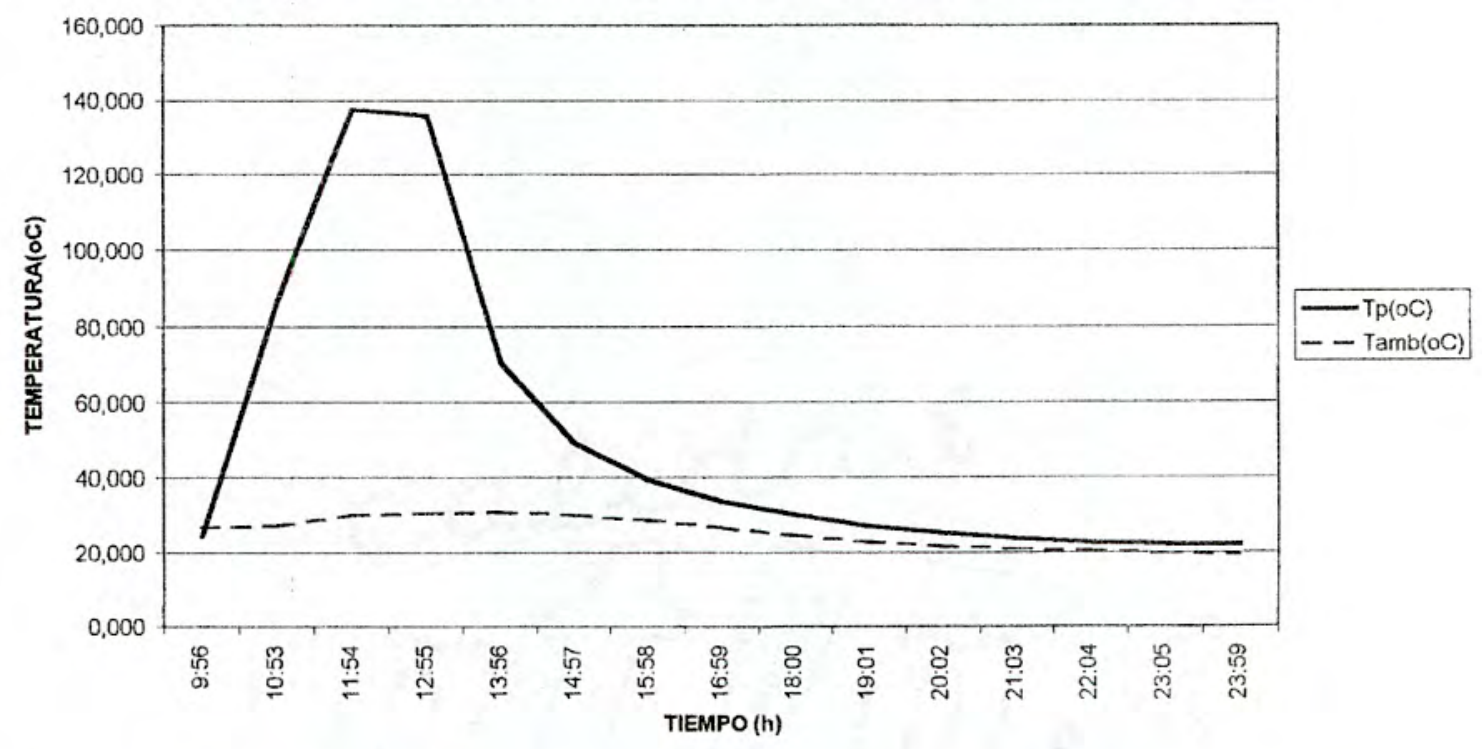

Tabla 3. Se muestran datos de temperaturas promedio horario en función del tiempo. Ta es la temperatura del agua dentro del recipiente y Tc es la temperatura dentro de la cámara de cocción. Las condiciones de operación son con un reflector y con carga, consistentes en un litro de agua.

\begin{tabular}{|c|c|c|c|c|c|c|c|c|c|}
\hline Dia & Tiempo(h) & $T p(o c)$ & $T a m b(o c)$ & $T c(o c)$ & $T a(o C)$ & $V v(m V)$ & $V v(m / s)$ & $G(m v)$ & $G\left(W / m^{2}\right)$ \\
\hline $07 / 03 / 00$ & $09: 49$ & 24.1 & 27 & 24.3 & 31.5 & 0.095 & 1.9 & 8.6 & 696.34 \\
\hline $07 / 03 / 00$ & $10: 49$ & 57.81 & 27.13 & 63.02 & 39.68 & 0.094 & 1.88 & 10.24 & 829.13 \\
\hline $07 / 03 / 00$ & $11: 50$ & 85.48 & 29.36 & 83.82 & 70.13 & 0.138 & 2.76 & 9.76 & 790.27 \\
\hline $07 / 03 / 00$ & $12: 52$ & 96.24 & 30.1 & 87.54 & 86.37 & 0.202 & 4.04 & 6.67 & 540.07 \\
\hline $07 / 03 / 00$ & $13: 53$ & 82.26 & 29.11 & 74 & 86.34 & 0.222 & 4.44 & 6.37 & 515.78 \\
\hline $07 / 03 / 00$ & $14: 53$ & 61.27 & 28.53 & 58.12 & 68.44 & 0.211 & 4.22 & 3.1 & 251.01 \\
\hline $07 / 03 / 00$ & $15: 53$ & 51.22 & 26.92 & 48.46 & 56.49 & 0.174 & 3.48 & 1.32 & 106.88 \\
\hline $07 / 03 / 00$ & $16: 53$ & 43.98 & 25.27 & 41.64 & 48.07 & 0.154 & 3.08 & 0.21 & 17.004 \\
\hline $07 / 03 / 00$ & $17: 53$ & 38.63 & 24.22 & 36.65 & 41.79 & 0.096 & 1.92 & 0 & 0 \\
\hline $07 / 03 / 00$ & $18: 53$ & 34.75 & 23.6 & 33.07 & 37.15 & 0.08 & 1.6 & 0 & 0 \\
\hline $07 / 03 / 00$ & $19: 53$ & 31.83 & 22.66 & 30.5 & 33.69 & 0.085 & 1.7 & 0 & 0 \\
\hline $07 / 03 / 00$ & $20: 53$ & 29.55 & 21.75 & 28.36 & 31.02 & 0.012 & 0.24 & 0 & 0 \\
\hline $07 / 03 / 00$ & $21: 53$ & 27.74 & 21.7 & 26.75 & 28.89 & 0.003 & 0.06 & 0 & 0 \\
\hline $07 / 03 / 00$ & $22: 53$ & 26.39 & 21.63 & 25.62 & 27.25 & 0.026 & 0.52 & 0 & 0 \\
\hline $07 / 03 / 00$ & $23: 53$ & 25.44 & 21.46 & 24.83 & 26.1 & 0.013 & 0.26 & 0 & 0 \\
\hline $07 / 03 / 00$ & $00: 53$ & 24.73 & 21.06 & 24.21 & 25.25 & 0.029 & 0.58 & 0 & 0 \\
\hline $07 / 03 / 00$ & $01: 53$ & 24.12 & 20.79 & 23.73 & 24.54 & 0.001 & 0.02 & 0 & 0 \\
\hline $07 / 03 / 00$ & $02: 53$ & 23.62 & 20.63 & 20.63 & 23.93 & 0.001 & 0.02 & 0 & 0 \\
\hline
\end{tabular}


Gráfica 3. Se muestra la variación de la temperatura de calentamiento/enfriamiento de la placa Tp, la temperatura de la cámara Tc $y$ de la temperatura del agua Ta, en la cocina/horno solar: Nos permite apreciar la diferencia de temperaturas entre la cámara y el agua.

TEMPERATURAS DE CALENTAMIENTO/ENFRIAMIENTO DE LA COCINA/HORNO SOLAR TIPO CAJA CON CARGA (T/07/03/00)

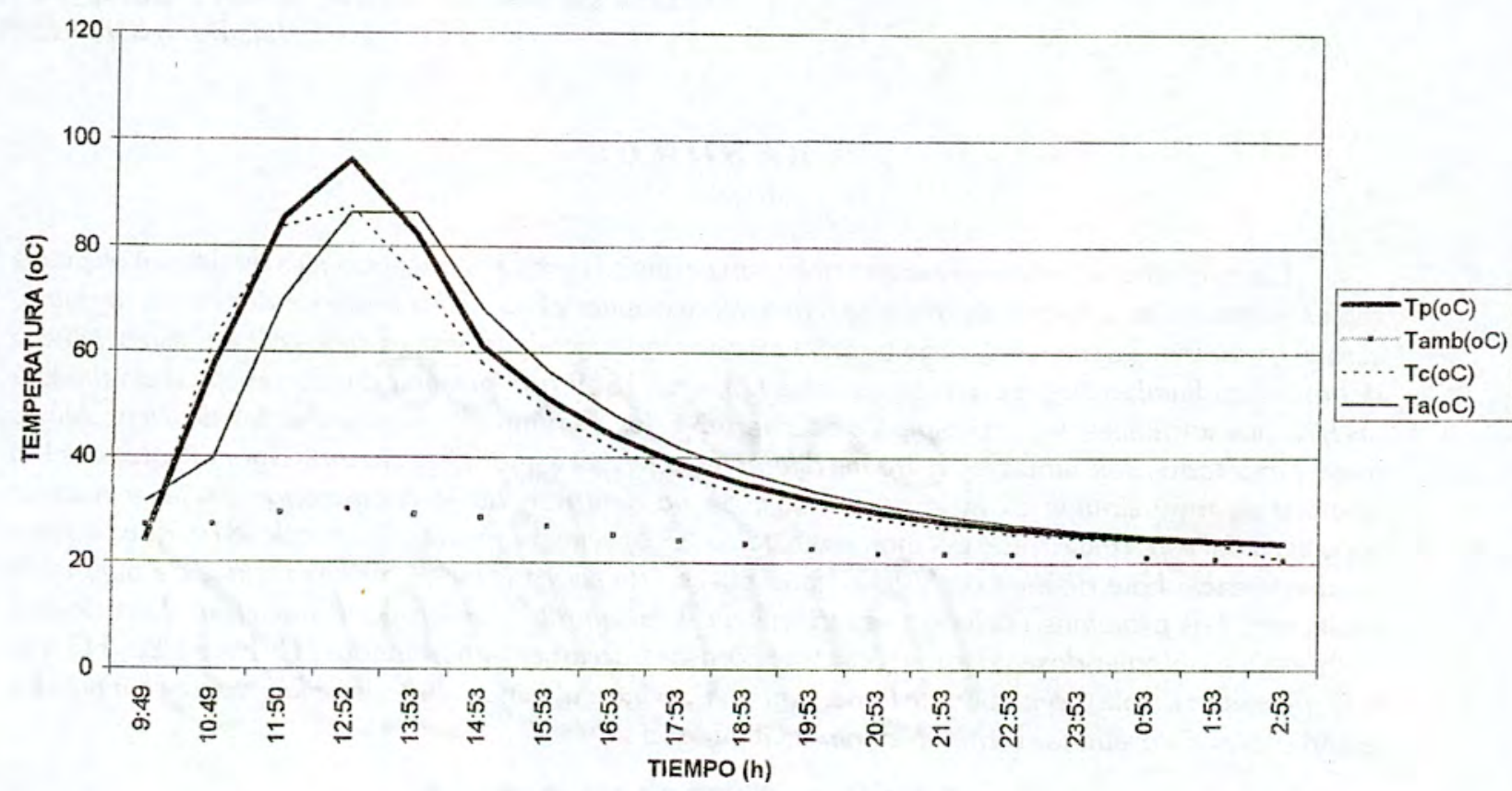

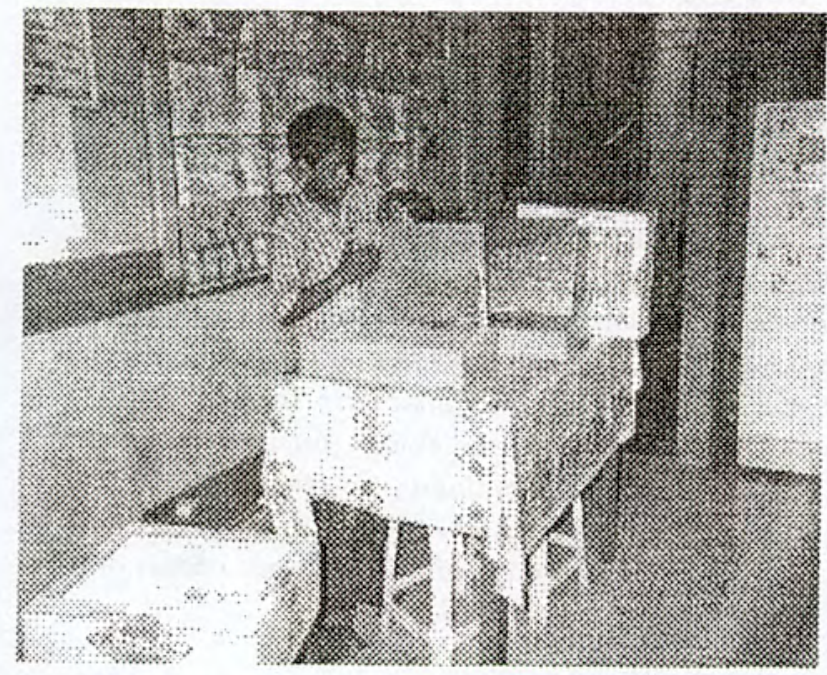

Foto 1. Proceso de construcción de una cocina solar tipo caja.

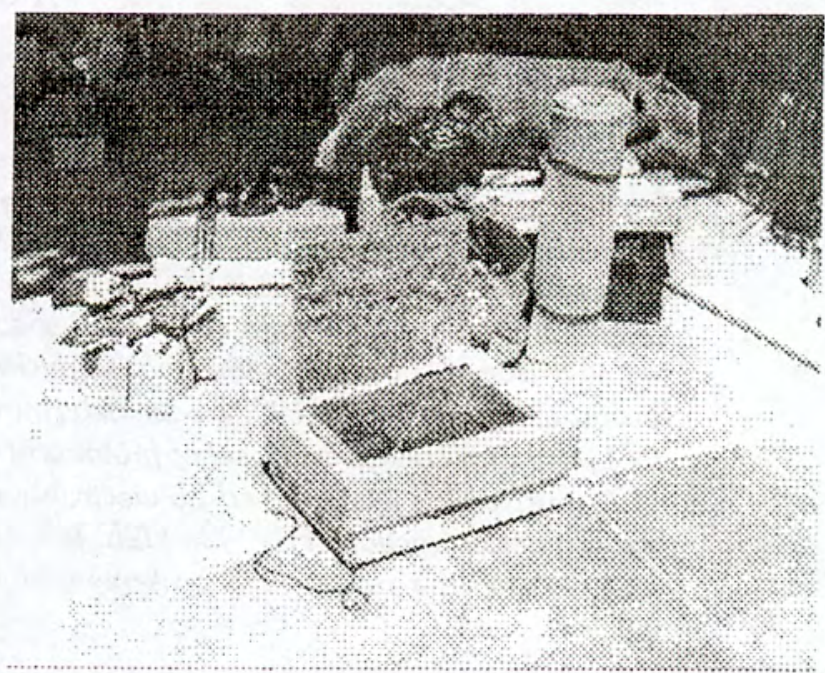

Foto 2. Modelo de cocina solar construida y evaluada. 